\title{
Business Process Modeling in Systems of Systems
}

\author{
Jean Marcos S. Santos', Valdemar Vicente Graciano Neto², Elisa Yumi Nakagawa ${ }^{1}$ \\ ${ }^{1}$ Institute of Mathematical and Computer Sciences - University of São Paulo \\ São Carlos - SP - Brazil \\ ${ }^{2}$ Institute of Informatics - Federal University of Goiás \\ Goiânia - GO - Brazil \\ jeanmarcos@usp.br, valdemarnetodinf.ufg.br, elisa@icmc.usp.br
}

\begin{abstract}
The Systems of Systems area has received more attention due to the growing demand from society and organizations for more integrated and complex services. As a result, the challenge for engineers to model business processes in increasingly complex systems has also grown. The main contribution of this paper is to provide a wide overview about the business process modeling approaches in the context of systems of systems. Through a systematic mapping of the literature, 33 studies were selected to answer four research questions. The analysis of the extracted data showed that the approaches to business process modeling has not evolve in the necessary way. This result shows that the area needs to receive more attention from researchers.
\end{abstract}

\section{Introduction}

Systems of Systems (SoS) are increasingly present within software engineering. SoS are alliances of independent systems (known as constituents) that work together to achieve complex behaviors. Several challenges arise in the development of such complex systems that address a set of unique characteristics when compared to other systems, i.e., the managerial and operational independence of constituents that leads to an often highly dynamic architecture. One of the challenges presented by SoS is its modeling, particularly for the business processes that rely on the interoperability of the constituents. SoS planning and architectural design stages can face difficulties. Once the architecture can be highly dynamic, the business process should be flexible and inter-organizational, which leads to problems with the precision of the current languages to precisely capture such aspects. Hence, we claim that current approaches and notations (e.g., UML, SySML and BPMN) do not provide a suitable support for complete modeling and adaptations as required [Neto et al. 2017]. This creates a gap in SoS modeling, which becomes a considerable obstacle if a Model-based Software Engineering (MBSE) approach is being used because it may not be possible to accurately represent the interactions and activities in that context.

To address this problem, this study used the guidelines prescribed by Kitchenham and Charters (2017) to conduct a Systematic Mapping and provide an overview of the use of languages and notations for business process modeling in SoS. From a set of 1400 retrieved studies, 33 were included and analyzed. Results reveal that the approaches to business process modeling has not evolve in the necessary way to fully serve the context of SoS.

The remainder of the paper is structured as follows. In the next section, the background is presented. Then, in Section 3, the methodology established for the conduction of the systematic mapping is introduced. In Section 4, the results from the analyses of 
collected data are presented and the research questions are answered and discussed. In Section 5, we present the main conclusions and limitations of this study.

\section{Background}

SoS are large-scale integrated systems that are heterogeneous and independently operable on their own but are networked together for a common goal [Jamshidi 2008].

Maier (1996) characterizes SoS using the following well-defined dimensions: (i) operational and managerial independence of the constituent systems, which can be independently managed and deliver functionalities even when not working with other systems; (ii) evolutionary development, once SoS may evolve over time to respond to changing characteristics in its environment, in its mission, or in the constituent systems; (iii) geographical distribution, because the systems collaborating in an SoS are distributed over a large geographic extent; they can only exchange information among themselves; (iv) emergent behaviors, which result from the collaboration of the constituent systems and cannot be achieved by any of the individual systems. The SoS architecture is then potentially dynamic, i.e., the architectural conformance changes over time due to autonomous constituents joining and leaving the SoS at runtime [Oquendo 2016].

Business Process Modeling (BPM) is the activity of representing processes of an enterprise. In software engineering, this modeling is often applied during the planning stage in mapping business requirements and workflows.

Several modeling languages can be used to facilitate the work of engineers in this task, such as Unified Modelling Language (UML), System Modeling Language (SysML), Business Process Model and Notation (BPMN) and others. These languages with their graphic notations facilitate communication and understanding of the processes, reducing possible ambiguities that would cause future side effects.

SoS, as traditional systems, also need to model their business processes, so the concepts and applications of BPM are also present. However, the unique characteristics of SoS make BPM more challenging. One of these issues, for example, is how to model collaborative processes between organizations when constituents can join or leave SoS at run time? [Neto et al. 2017]. The study reported herein then aims to spot that subject.

\section{Methodology}

This study conducted a literature search by performing a secondary research called the Systematic Mapping Study. This type of study is designed to provide a wide overview of a research area to establish if research evidence exists on a topic and provide an indication of the quantity of the evidence [Kitchenham and Charters 2007]. Petersen, Vakkalanka, and Kuzniarz (2015) defines the Systematic Mapping Study as a defined method to build a classification scheme and structure a software engineering field of interest.

A Systematic Mapping Study has the same rigor of a Systematic Literature Review (SLR) and needs to follow the same steps: (i) planning, (ii) conducting, (iii) reporting. The planning stage will support the researchers to define, among other things, the scope of the study, the research questions and strategy, as well as the inclusion and exclusion criteria and the data extraction form. In the conduction stage, the defined plan will be executed, and the studies will be searched, evaluated, selected and the relevant information are extracted so that the research questions can be answered. Finally, in the reporting stage, the results of the 
review will be disseminated. Academics usually assume that dissemination is about reporting results in academic journals and / or conferences.

\subsection{Protocol}

The protocol summary of this systematic mapping study is shown in Table 1. Details of the protocol are available in https://encurtador.com.br/hwyP4.

Table 1 - Protocol Summary.

\begin{tabular}{|c|c|}
\hline Research Questions & $\begin{array}{l}\text { RQ1: What notations have been used to specify and manage business } \\
\text { processes in the context of Systems of Systems? } \\
\text { Rationale: Understanding what notations are being used to specify and } \\
\text { manage business processes provides a way to gain a broader view of } \\
\text { BPM activity in SoS, given the importance of notations for the activity. } \\
\text { RQ2: Which difficulties related to business processes have been faced in } \\
\text { Systems of Systems? } \\
\text { Rationale: This RQ brings information about the existing problems } \\
\text { related to business processes in systems of systems. This information } \\
\text { can guide the search for new solutions and improvements in the area. } \\
\text { RQ3: How has Business Process Model and Notation been applied in the } \\
\text { context of Systems of Systems? } \\
\text { Rationale: Due to the importance of BPMN in the business process, it is } \\
\text { important to detail its use and its limitations when applied in SoS. } \\
\text { RQ4: Are the notations used to model business process in Systems of } \\
\text { Systems expressive enough for representing all Systems of Systems } \\
\text { characteristics? } \\
\text { Rationale: This RQ collects information about the coverage of the } \\
\text { notations currently used to model business processes, considering that } \\
\text { the characteristics of SoS tend to be more complex, and that information } \\
\text { can answer if there is a need for new symbols or notations to support } \\
\text { SoS. }\end{array}$ \\
\hline Search String & $\begin{array}{l}\text { ("systems of systems" OR "SoS" OR "system of system" OR "systems of } \\
\text { system" OR "system of systems" OR "systems-of-systems" OR "system- } \\
\text { of-system" OR "systems-of-system" OR "system-of-systems" OR } \\
\text { "systems of information systems" OR "SolS" OR "systems of information } \\
\text { system" OR "systems-of-information systems" OR "systems-of- } \\
\text { information system") AND ("business process modeling" OR "BPM" OR } \\
\text { "business process modelling" OR "business process model" OR "BPMN") }\end{array}$ \\
\hline Search Strategy & $\begin{array}{l}\text { Scopus; IEEExplore; ACM Digital Library; Google Scholar; Engineering } \\
\text { Village; SpringerLink }\end{array}$ \\
\hline Inclusion Criteria & $\begin{array}{l}\text { IC1: The study addresses the use of Business Processes in Systems of } \\
\text { Systems } \\
\text { IC2: The study addresses the use of Business Processes in Systems-of- } \\
\text { Information }\end{array}$ \\
\hline Exclusion Criteria & $\begin{array}{l}\text { EC1: The study does not address the use of Business Process in } \\
\text { Systems of Systems } \\
\text { EC2: The study does not address the use of Business Process in } \\
\text { Systems-of-Information Systems } \\
\text { EC3: The study is written in a language other than English } \\
\text { EC4: The full text of the study is not available } \\
\text { EC5: The study is directly related to another primary study of the same } \\
\text { author } \\
\text { EC6: The study was not peer-reviewed } \\
\text { EC7: The study is gray literature }\end{array}$ \\
\hline
\end{tabular}




\begin{tabular}{|l|l|}
\hline & QQ1: Do the authors present an overview of related work and \\
& background regarding the area of the study? \\
QQ2: Does the study provide a clear justification about the methods used \\
Quality Assessment & in the study? \\
& QQ3: Is there a clear statement of contributions and has sufficient data \\
& been presented to support them? \\
& QQ4: Do the authors explicitly discuss the credibility and limitations of \\
& their findings?
\end{tabular}

\section{Results}

Table 2 shows the results of the search, and the application of the inclusion and exclusion criteria. The list of all selected studies and their respective identifiers can be found in https://encurtador.com.br/hwyP4.

Table 2 - Databases with total, excluded and included studies.

\begin{tabular}{|l|l|l|l|l|}
\hline \multicolumn{1}{|c|}{ Database } & \multicolumn{1}{c|}{ Total } & \multicolumn{1}{c|}{ Duplicated } & \multicolumn{1}{c|}{ Excluded } & Included \\
\hline Scopus & 471 & 2 & 440 & 29 \\
\hline IEEExplore & 19 & 18 & 1 & 0 \\
\hline ACM Digital Library & 186 & 25 & 160 & 1 \\
\hline Google Scholar & 100 & 32 & 65 & 3 \\
\hline Engineering Village & 44 & 39 & 5 & 0 \\
\hline SpringerLink & 580 & 84 & 496 & 0 \\
\hline Total & $\mathbf{1 4 0 0}$ & $\mathbf{2 0 0}$ & $\mathbf{1 1 6 7}$ & $\mathbf{3 3}$ \\
\hline
\end{tabular}

Figure 1 shows the 33 selected studies distribution over years. We can see an increase in interest between BPM and SoS as of 2016, but it still seems to be too small and fluctuating to be able to face all the challenges that these complex systems address.

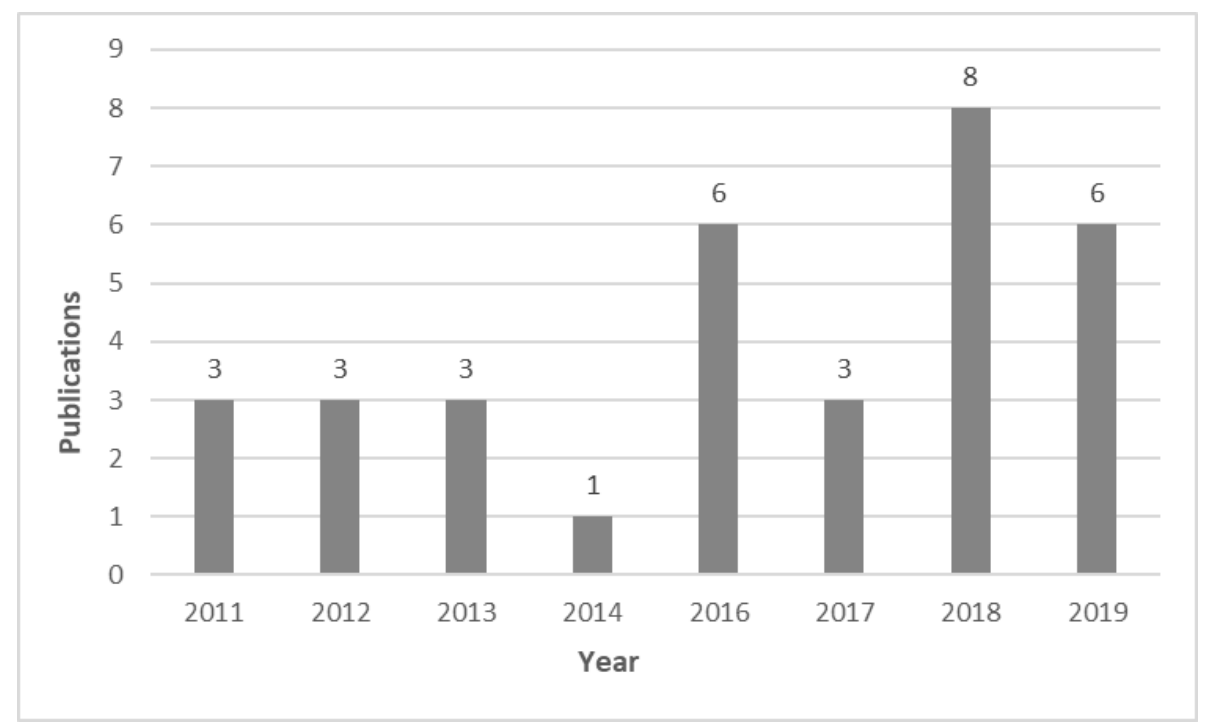

Fig 1. Publications per year.

RQ1: What notations have been used to specify and manage business processes in the context of Systems of Systems?

Figure 2 shows the models/languages/notations that were used or presented as an option in the studies. We can see that BPMN has a wide adoption in the SoS area, but not necessarily to model only business processes. Most studies that used BPMN, did it to model parts of the 
architecture, but without addressing all the characteristics of SoS. Only $6 \%$ of the studies (Lahboube, Roudies, and Souissi 2016; Wu et al. 2012) explicitly mention the modeling of business processes in SoS. This may indicate the lack of a notation that supports the full representation of business processes in SoS.

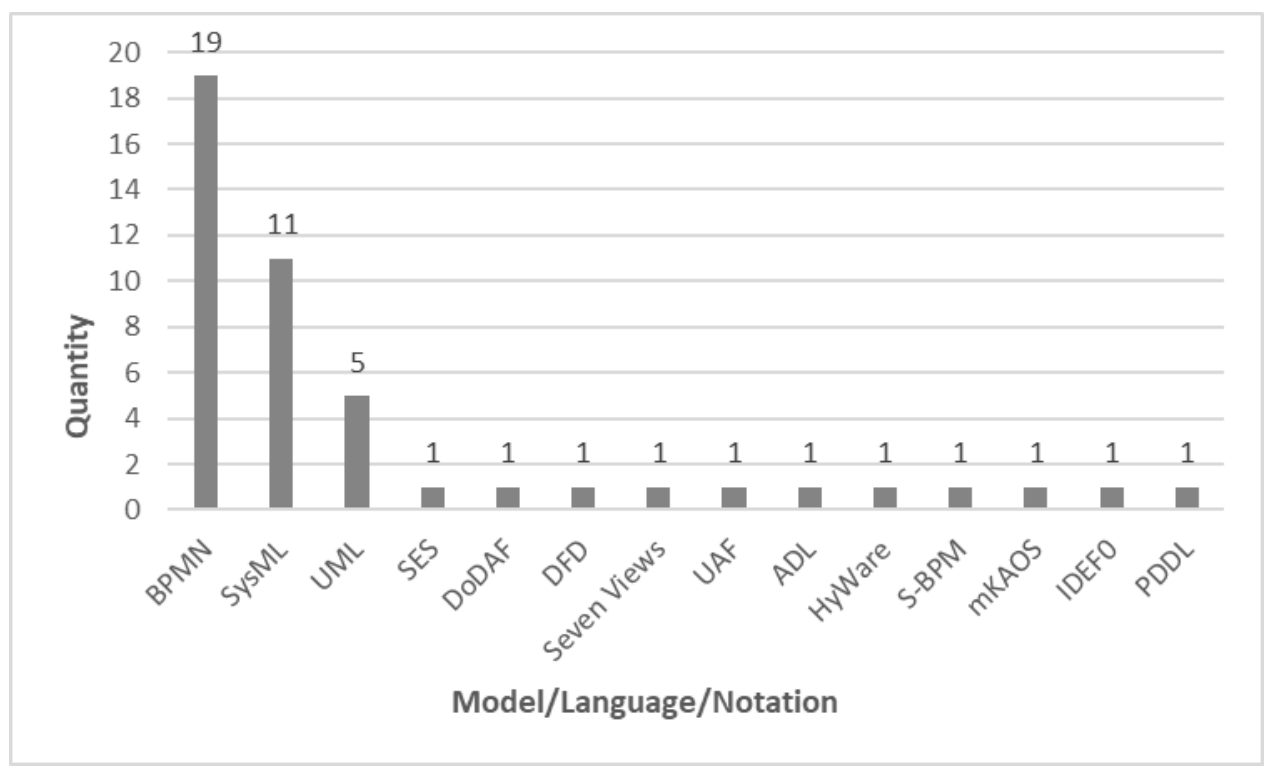

Fig 2. Models/languages/notations presented in the selected studies.

RQ2: Which difficulties related to business processes have been faced in Systems of Systems?

The biggest problem reported by the studies refers to the lack of expressiveness of the elements present in the notations [Arnautovic, Svetinovic and Diabat 2012; Neto et al. 2016; Tikito and Souissi 2017; Neto et al. 2018], mainly to represent the interactions between the systems and thus be able to represent the emerging behaviors.

The analysis of the studies also demonstrated the lack of notations and tools that could execute the defined models without the need to transform a non-executable language as UML and BPMN to an executable one such as BPEL or DEVS (S22, S26, S9).

This transformation step to allow the execution of the models could be avoided, if there were alternatives to directly execute the models created with BPMN. However, none of the studies demonstrated that this type of direct execution is possible. In Candela et al. (2017) (S19) this problem does not happen because the language presented for modeling is also an executable language.

RQ3: How has Business Process Model and Notation been applied in the context of Systems of Systems?

BPMN was present in $58 \%$ of the studies (S28, S11, S12, S1, S4, S14, S17, S18, S15, S21, S22, S23, S9, S3, S10, S6, S25, S26, S27), showing great adoption in the context of SoS. This is largely due to its popularity that already exists in normal software engineering contexts. 


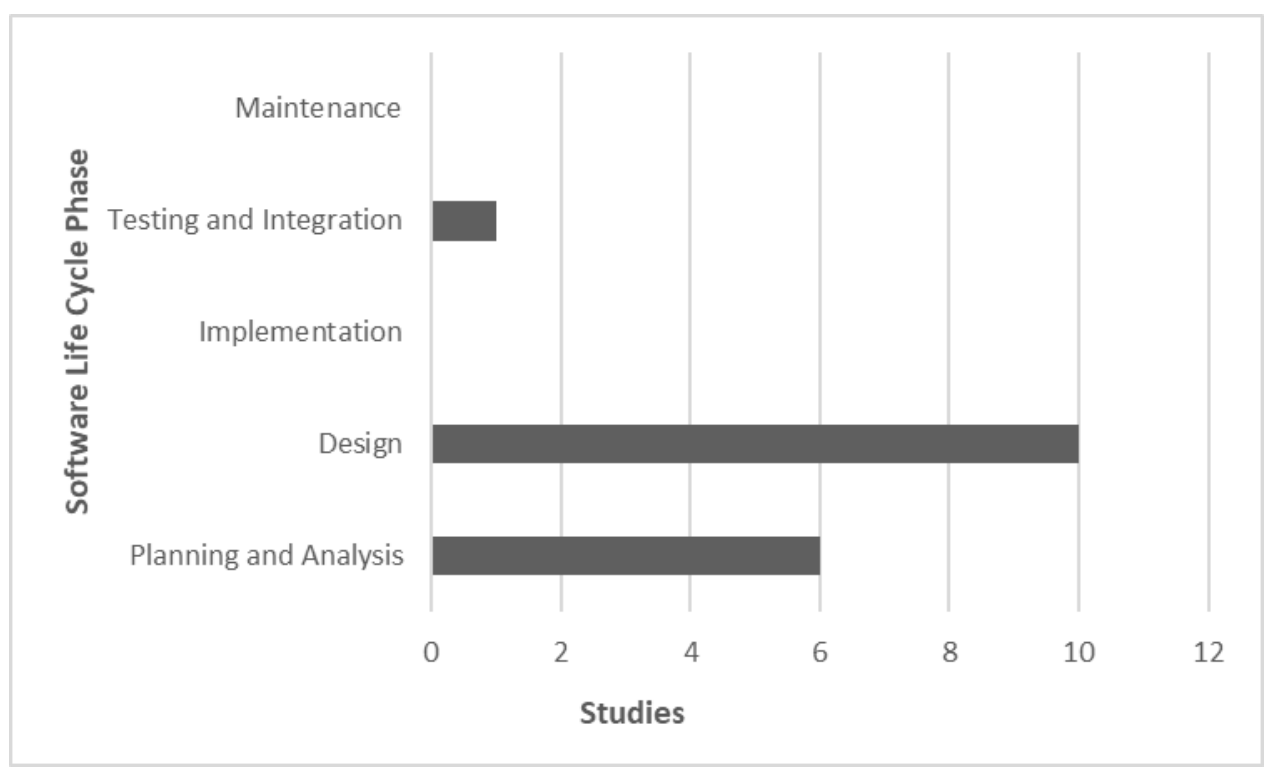

Fig 3. BPMN usage in software life cycle phases.

The analysis of the studies showed that despite the real purpose of BPMN being the modeling of business processes, it is more applied in the design stage, than in the planning and analysis stage. One of the reasons for this result may be the BPMN's inability to cover all the characteristics of SoS modeling, a problem that was found by this systematic mapping. Another reason is that BPMN is a trivial and easy to understand notation, which is why it was used to model parts of the system architecture that did not require a formalism, such as, for example, to model the understanding of some aspect or solution (S28).

\section{RQ4: Are the notations used to model business processes in Systems of Systems expressive enough for representing all Systems of Systems characteristics?}

The synthesis of the extracted data shows us that the notations used to model business processes in SoS are not yet sufficiently mature and adequate to meet the special needs of this context. Some studies (S1, S29, S20) indicated that modifications are necessary to achieve greater representativeness, either by modifying existing notations or creating new ones.

Another strong indication that supports this observation is the low number of studies that directly address the theme or that cites the need to adapt the characteristics of SoS. The challenges of modeling business processes still seem to be too abstract to be portrayed directly in studies in the area.

When BPMN was the language chosen to model some aspects of SoS, considerations were cited about how it could better serve the context of SoS, such as providing easier ways to represent the interactions between SoS component systems (S17); more dynamic characteristics that make it possible to represent the emerging behaviors of SoS (S15); better ways to model processes with complex data dependencies (S26).

\subsection{Quality Assessment}

The selected studies were evaluated on each of the quality questions. For each question, 3 notes were possible according to the following scale: (i) the study fully meets a given quality criterion (1.0 point); (ii) the study meets the quality criterion to some extent ( 0.5 point); and (iii) the study does not meet a quality criterion ( 0 point). Table 3 shows the result of the assessment. 
The quality assessment showed that $75 \%$ of the selected studies had an assessment above 2.5 , which is a good indication of the quality of the studies since the highest value on the scale is 4 .

Table 3 - Quality assessment of selected studies.

\begin{tabular}{|l|l|l|l|}
\hline$\#$ & Quality Score & $\#$ & Quality Score \\
\hline S23 & 4.0 & S8 & 3.0 \\
\hline S22 & 4.0 & S16 & 2.5 \\
\hline S29 & 4.0 & S25 & 2.5 \\
\hline S20 & 4.0 & S11 & 2.5 \\
\hline S26 & 4.0 & S27 & 2.5 \\
\hline S30 & 4.0 & S32 & 2.5 \\
\hline S31 & 3.5 & S6 & 2.5 \\
\hline S9 & 3.0 & S5 & 2.5 \\
\hline S15 & 3.0 & S4 & 2.0 \\
\hline S10 & 3.0 & S17 & 2.0 \\
\hline S14 & 3.0 & S2 & 2.0 \\
\hline S19 & 3.0 & S21 & 2.0 \\
\hline S18 & 3.0 & S13 & 2.0 \\
\hline S24 & 3.0 & S28 & 2.0 \\
\hline S33 & 3.0 & S1 & 1.0 \\
\hline S3 & 3.0 & S12 & 0.5 \\
\hline S7 & 3.0 & & \\
\hline
\end{tabular}

\subsection{Threats to Validity}

There are some threats to validity in the findings presented in this study. As the research area that this study addresses is new, it was not possible to define any control group to calibrate the search string. Due to these facts, important studies may have been left out of the selection. To minimize this possibility, six databases were included, and the search string was created with many combinations of synonyms.

More threats to validity are present in the data extraction process to answer the research questions, it was necessary to subjectively interpret the information provided by the studies, since many studies do not clearly expose details about the investigated questions. To mitigate this possible problem, the data extraction was reviewed by an expert.

\section{Final Remarks}

This study presented a systematic mapping study on Business Process Modeling in the context of Systems of Systems, with special emphasis on the notations used in modeling. As we have seen, there is still a lot of work to be done in the area.

The contributions of this study include the realization of the need for more studies that address the challenges of BPM in SoS, so that the area can be better analyzed by more researched, more problems and challenges can be recognized and solutions and improvements can be developed.

As a future work, we point out the need to define a notation for the modeling of business processes that can represent all the characteristics of SoS, including the interactions between the constituent systems and the emerging behaviors that arise from their interactions.

\section{References}

M. Maier, (1996) “Architecting Principles for Systems-of- Systems”, INCOSE International Symposium, vol. 6, pp. 565-573. 
M. Chaabane, I. B. Rodriguez, R. Colomo-Palacios, W. Gaaloul, M. Jmaiel (2019) “A modeling approach for Systems-of-Systems by adapting ISO/IEC/IEEE 42010 Standard evaluated by Goal-Question-Metric", Science of Computer Programming, Volume 184, 2019.

V. V. G. Neto, E. Cavalcante, J. El Hachem, D. S. Santos, (2017) "On the Interplay of Business Process Modeling and Missions in Systems-of-Information Systems", IEEE/ACM Joint 5th International Workshop on Software Engineering for Systems-ofSystems and 11th Workshop on Distributed Software Development, Software Ecosystems and Systems-of-Systems (JSOS), Buenos Aires, 2017, pp. 72-73.

B. Kitchenham, S. Charters, (2007) "Guidelines for performing systematic literature reviews in software engineering”, Technical report, EBSE-2007-01, School of Comp. Science and Mathematics, Keele University.

M. Jamshidi, (2008) "Systems of Systems Engineering: Principles and Applications”, CRC Press Taylor \& Francis Group, FL, USA.

K. Petersen, S. Vakkalanka, L. Kuzniarz (2015) "Guidelines for conducting systematic mapping studies in software engineering: An update", Information and Software Technology, vol. 64, pp. 1-18.

L. Garces, A. Ampatzoglou, P. Avgeriou, and E. Y. Nakagawa, (2017) “Quality attributes and quality models for ambient assisted living software systems: A systematic mapping" Information and Software Technology, vol. 82, pp. 121-138.

P. G. Teixeira, V. H. L. Lopes, R. Pereira dos Santos, M. Kassab and V. V. Graciano Neto, (2019) "The Status Quo of Systems-of-Information Systems" 2019 IEEE/ACM 7th International Workshop on Software Engineering for Systems-of-Systems (SESoS) and 13th Workshop on Distributed Software Development, Software Ecosystems and Systems-of-Systems (WDES), Montreal, QC, Canada, pp. 34-41.

Y. Wu, R. Kang, X. Pan, J. Yang, (2012) "The assessment of sos's structure based on the MCSP" Advanced Materials Research, vol 472-475, pp. 3188-3193.

F. Lahboube, O. Roudies, N. Souissi, (2016) "Seven views + one" Proceedings of 2016 International Conference on Electrical and Information Technologies, ICEIT, pp. 55-60.

E. Arnautovic, D. Svetinovic, A. Diabat, (2012) "Business interactions modeling for systems of systems engineering: Smart grid example" Proceedings - 2012 7th International Conference on System of Systems Engineering, SoSE, Genova, 2012, pp. 107-112.

V. V. G. Neto et al. (2016) "Experience report and challenges for systems-of-systems engineering: a real case in the Brazilian defense domain" Proceedings of Workshop on Distributed Development, Software Ecosystems and Systems of Systems, Maringá, Brazil, pp. 41-50

I. Tikito, N. Souissi, (2017) "Data collect requirements model" 2nd international Conference on Big Data, Cloud and Applications (BDCA'17). Association for Computing Machinery, New York, NY, USA, Article 4, 1-7.

V. V. G. Neto et al. (2018) "A study on goals specification for systems-of-information systems: Design principles and a conceptual model" XIV Brazilian Symposium on Information Systems (SBSI'18), Caxias do Sul, Brazil. Article 21, 1-8. ACM.

L. Candela, F. Giannotti, V. Grossi, P. Manghi, R. Trasarti, (2017) "HyWare: A hybrid workflow language for research E-infrastructures" D-Lib Magazine, vol 23, number 1/2. 
F. Oquendo "Formally describing the software architecture of Systems-of-Systems with SosADL" 11 th System of Systems Engineering Conference (SoSE), Kongsberg, 2016, pp. 1-6. IEEE.

\section{Appendix A - Selected Studies}

\begin{tabular}{|c|c|c|c|}
\hline \# & Title & Author & Year \\
\hline S1 & Architecture evolution and evaluation (ArchEE) capability & Jain, $P$. & 2011 \\
\hline S2 & $\begin{array}{l}\text { Model based systems engineering for smart grids as systems of } \\
\text { systems }\end{array}$ & Lopes, Amit $\mathrm{J}$ and Lezama, R and Pineda, Ricardo & 2011 \\
\hline S3 & Modeling and simulation for SoS based on the DoDAF framework & Pan, $\mathrm{X}$. and Yin, B. and $\mathrm{Hu}, \mathrm{J}$. & 2011 \\
\hline S4 & $\begin{array}{l}\text { Business interactions modeling for systems of systems engineering: } \\
\text { Smart grid example }\end{array}$ & Arnautovic, E. and Svetinovic, D. and Diabat, A. & 2012 \\
\hline S5 & Model-based requirements engineering for system of systems & $\begin{array}{l}\text { Holt, J. and Perry, S. and Brownsword, M. and } \\
\text { Cancila, D. and Hallerstede, S. and Hansen, F.O. }\end{array}$ & 2012 \\
\hline S6 & The assessment of sos's structure based on the MCSP & Wu, Y. and Kang, R. and Pan, X. and Yang, J. & 2012 \\
\hline S7 & Fault modelling for systems of systems & $\begin{array}{l}\text { Andrews, Z. and Fitzgerald, J. and Payne, R. and } \\
\text { Romanovsky, A. }\end{array}$ & 2013 \\
\hline S8 & Model-based development of fault tolerant systems of systems & $\begin{array}{l}\text { Andrews, Z. and Payne, R. and Romanovsky, A. } \\
\text { and Didier, A. and Mota, A. }\end{array}$ & 2013 \\
\hline S9 & $\begin{array}{l}\text { Model-Driven Systems Engineering for Netcentric System of Systems } \\
\text { with DEVS Unified Process }\end{array}$ & Mittal, Saurabh and Martín, José Luis Risco & 2013 \\
\hline S10 & Processing chains in system of systems & $\begin{array}{l}\text { Ploom, T. and Last, I. and Glaser, A. and Scheit, } \\
\text { S. }\end{array}$ & 2014 \\
\hline S11 & $\begin{array}{l}\text { A requirements engineering and management process in concept } \\
\text { phase of complex systems }\end{array}$ & $\begin{array}{l}\text { Arnaut, B.M. and Ferrari, D.B. and De Oliveira E } \\
\text { Souza, M.L. }\end{array}$ & 2016 \\
\hline S12 & $\begin{array}{l}\text { An iterative and recursive model-based system of systems engineering } \\
\text { (MBSoSE) approach for product development in the medical device } \\
\text { domain }\end{array}$ & Ciancia, P. & 2016 \\
\hline S13 & Building a HIS supervision metamodel & Lahboube, F. and Roudies, O. and Souissi, N. & 2016 \\
\hline S14 & $\begin{array}{l}\text { Contributing to the GEO Model Web implementation: A brokering } \\
\text { service for business processes }\end{array}$ & Santoro, M. and Nativi, S. and Mazzetti, P. & 2016 \\
\hline S15 & $\begin{array}{l}\text { Experience report and challenges for systems-of-systems engineering: } \\
\text { a real case in the Brazilian defense domain }\end{array}$ & $\begin{array}{l}\text { Paes, Carlos Eduardo de B and Neto, VV } \\
\text { Graciano and Oquendo, Flávio and Nakagawa, } \\
\text { Elisa Yumi and Carlos--SP--Brazil, Sao }\end{array}$ & 2016 \\
\hline S16 & Seven views + one & Lahboube, F. and Roudies, O. and Souissi, N. & 2016 \\
\hline S17 & Data collect requirements model & Tikito, I. and Souissi, N. & 2017 \\
\hline S18 & $\begin{array}{l}\text { Engineering systems by combining BPMN and HLA-based distributed } \\
\text { simulation }\end{array}$ & $\begin{array}{l}\text { Falcone, A. and Garro, A. and Daambrogio, A. and } \\
\text { Giglio, A. }\end{array}$ & 2017 \\
\hline S19 & HyWare: A hybrid workflow language for research E-infrastructures & $\begin{array}{l}\text { Candela, L. and Giannotti, F. and Grossi, V. and } \\
\text { Manghi, P. and Trasarti, R. }\end{array}$ & 2017 \\
\hline S20 & $\begin{array}{l}\text { A study on goals specification for systems-of-information systems: } \\
\text { Design principles and a conceptual model }\end{array}$ & $\begin{array}{l}\text { Graciano Neto, V.V. and Rohling, A. and Horita, F. } \\
\text { and El-Hachem, J. and Cavalcante, E. and Santos, } \\
\text { D. and Nakagawa, E.Y. }\end{array}$ & 2018 \\
\hline S21 & $\begin{array}{l}\text { Graphical user interface definition processes in the frame of systems- } \\
\text { of-systems }\end{array}$ & Arnould, V. & 2018 \\
\hline S22 & $\begin{array}{l}\text { Including co-simulation in modeling and simulation tool for supporting } \\
\text { risk management in industrial context }\end{array}$ & $\begin{array}{l}\text { Gorecki, S. and Bouanan, Y. and Ribault, J. and } \\
\text { Zacharewicz, G. and Perry, N. }\end{array}$ & 2018 \\
\hline S23 & $\begin{array}{l}\text { Mandala: An agent-based platform to support interoperability in } \\
\text { systems-of-systems }\end{array}$ & $\begin{array}{l}\text { Mendes, A. and Loss, S. and Cavalcante, E. and } \\
\text { Lopes, F. and Batista, T. }\end{array}$ & 2018 \\
\hline S24 & Representing processes of human robot collaboration & Weichhart, G. & 2018 \\
\hline S25 & $\begin{array}{l}\text { The logic of information and processes in system-of-systems } \\
\text { applications }\end{array}$ & Eklund, P. and Johansson, M. and Kortelainen, J. & 2018 \\
\hline S26 & $\begin{array}{l}\text { Using bpmn and HLA for sos engineering: lessons learned and future } \\
\text { directions }\end{array}$ & $\begin{array}{l}\text { Falcone, A. and Garro, A. and Drambrogio, A. and } \\
\text { Giglio, A. }\end{array}$ & 2018 \\
\hline S27 & $\begin{array}{l}\text { Using model-driven approach for engineering the system engineering } \\
\text { system }\end{array}$ & Arnould, V. & 2018 \\
\hline S28 & A BPMN/HLA-Based Methodology for Collaborative Distributed des & $\begin{array}{l}\text { Possik, J. and Amrani, A. and Vallespir, B. and } \\
\text { D'Ambrogio, A. and Zacharewicz, G. }\end{array}$ & 2019 \\
\hline S29 & $\begin{array}{l}\text { A modeling approach for Systems-of-Systems by adapting } \\
\text { ISO/IEC/IEEE } 42010 \text { Standard evaluated by Goal-Question-Metric }\end{array}$ & $\begin{array}{l}\text { Chaabane, Mariam and Rodriguez, Ismael } \\
\text { Bouassida and Colomo-Palacios, Ricardo and } \\
\text { Gaaloul, Walid and Jmaiel, Mohamed }\end{array}$ & 2019 \\
\hline S30 & A perceptive interface for intelligent cyber enterprises & $\begin{array}{l}\text { Dumitrache, I. and Caramihai, S.I. and Moisescu, } \\
\text { M.A. and Sacala, I.S. and Vladareanu, L. and } \\
\text { Repta, D. }\end{array}$ & 2019 \\
\hline S31 & $\begin{array}{l}\text { Designing development processes related to system of systems using } \\
\text { a modeling framework }\end{array}$ & Shaked, A. and Reich, Y. & 2019 \\
\hline
\end{tabular}


